\title{
A REMARK ON FOUR-DIMENSIONAL ALMOST KÄHLER-EINSTEIN MANIFOLDS WITH NEGATIVE SCALAR CURVATURE
}

\author{
R. S. LEMENCE, T. OGURO, and K. SEKIGAWA
}

Received 12 October 2003

Concerning the Goldberg conjecture, we will prove a result obtained by applying the result of Iton in terms of $L^{2}$-norm of the scalar curvature.

2000 Mathematics Subject Classification: 53C25, 53C55.

1. Introduction. An almost Hermitian manifold $M$ is called an almost Kähler manifold if the corresponding Kähler form is a closed 2-form. It is well known that an almost Kähler manifold with integrable almost-complex structure is Kählerian. Concerning the integrability of almost Kähler manifold, the following conjecture by Goldberg is known (see [2]).

CONJECTURE 1.1. A compact almost Kähler-Einstein manifold is Kählerian.

Sekigawa [8] proved that the conjecture is true if the scalar curvature $\tau$ of $M$ is nonnegative. But the conjecture is still open in the case where $\tau$ is negative. Recently, applying the Seiberg-Witten theory, Itoh [4] obtained the following integrability condition for certain almost Kähler-Einstein 4-manifolds in terms of the $L^{2}$-norm of the scalar curvature.

THEOREM 1.2 [4]. Let $M$ be a four-dimensional compact almost Kähler-Einstein manifold with negative scalar curvature. If $M$ satisfies

$$
\int_{M} \tau^{2} d V=32 \pi^{2}\left(2 \chi(M)+p_{1}(M)\right)
$$

then it must be Kähler-Einstein. Here, $\chi(M)$ and $p_{1}(M)$ are the Euler characteristic and the first Pontrjagin number of $M$, respectively.

As a corollary, he also proved the following.

COROLlary 1.3 [4]. Let $M$ be a four-dimensional compact almost Kähler-Einstein manifold with negative scalar curvature. If $M$ satisfies

$$
\int_{M} \tau^{2} d V \leq 24 \int_{M}\left\|W^{+}\right\|^{2} d V
$$

or, more strictly, if $|\tau| \leq 2 \sqrt{6}\left\|W^{+}\right\|$at each point of $M$, then $M$ must be Kähler-Einstein. Here, $\mathfrak{W}^{+}$is the self-dual Weyl curvature operator of the metric $g$. 
In this paper, concerning the Goldberg conjecture, we will prove a result obtained by using Corollary 1.3 (see Theorem 2.2).

2. Preliminaries and the result. Let $M=(M, J, g)$ be a four-dimensional almost Kähler-Einstein manifold with the almost-complex structure $J$ and the Hermitian metric $g$. We denote by $\Omega$ the Kähler form of $M$ defined by $\Omega(X, Y)=g(X, J Y)$ for $X, Y \in \mathfrak{X}(M)$, the set of all smooth vector fields on $M$. We assume that $M$ is oriented by the volume form $d V=\Omega^{2} / 2$. We denote by $\nabla, R, \rho$, and $\tau$ the Riemannian connection, the curvature tensor, the Ricci tensor, and the scalar curvature of $M$, respectively. We assume that the curvature tensor is defined by $R(X, Y) Z=\left[\nabla_{X}, \nabla_{Y}\right] Z-\nabla_{[X, Y]} Z$ for $X, Y, Z \in \mathfrak{X}(M)$. We denote by $\rho^{*}$ the Ricci $*$-tensor of $M$ defined by

$$
\rho^{*}(x, y)=\frac{1}{2} \text { trace of }(z \longmapsto R(x, J y) J z)
$$

for $x, y, z \in T_{p} M$, the tangent space of $M$ at $p \in M$. The Ricci $*$-tensor satisfies $\rho^{*}(x$, $y)=\rho^{*}(J y, J x)$ for any $x, y \in T_{p} M, p \in M$. We note that if $M$ is Kählerian, the Ricci tensor and the Ricci $*$-tensor coincide on $M$. The $*$-scalar curvature $\tau^{*}$ of $M$ is the trace of the linear endomorphism $Q^{*}$ defined by $g\left(Q^{*} x, y\right)=\rho^{*}(x, y)$ for $x, y \in T_{p} M$, $p \in M$. Since $\|\nabla J\|^{2}=2\left(\tau^{*}-\tau\right), M$ is a Kähler manifold if and only if $\tau^{*}-\tau=0$ on $M$. An almost Hermitian manifold $M$ is called a weakly $*$-Einstein manifold if $\rho^{*}=\lambda^{*} g\left(\lambda^{*}=\right.$ $\left.\tau^{*} / 4\right)$ and a $*$-Einstein if $M$ is weakly $*$-Einstein with constant $*$-scalar curvature. The following identity holds for any four-dimensional almost Hermitian Einstein manifold:

$$
\frac{1}{2}\left\{\rho^{*}(x, y)+\rho^{*}(y, x)\right\}=\frac{\tau^{*}}{4} g(x, y)
$$

for $x, y \in T_{p} M, p \in M$.

Now, let $\wedge^{2} M$ be the vector bundle of all real 2-forms on $M$. The bundle $\wedge^{2} M$ inherits a natural inner product $g$ and we have an orthogonal decomposition

$$
\wedge^{2} M=\mathbb{R} \Omega \oplus L M \oplus \wedge_{0}^{1,1} M
$$

where $L M$ (resp., $\wedge_{0}^{1,1} M$ ) is the bundle of $J$-skew-invariant (resp., $J$-invariant) 2-forms on $M$ perpendicular to $\Omega$. We can identify the subbundle $\mathbb{R} \Omega \oplus L M$ (resp., $\wedge_{0}^{1,1} M$ ) with the bundle $\wedge_{+}^{2} M$ (resp., $\wedge_{-}^{2} M$ ) of self-dual (resp., anti-self-dual) 2-forms on $M$. Since $M$ is Einstein, it is well known that the curvature operator $\mathscr{R}: \wedge^{2} M \rightarrow \wedge^{2} M$ preserves $\wedge_{ \pm}^{2} M$ and that the Weyl curvature operator $\mathcal{W}: \wedge^{2} M \rightarrow \wedge^{2} M$ is given by

$$
\mathscr{W}(\iota(X) \wedge \iota(Y))=\mathscr{R}(\iota(X) \wedge \iota(Y))-\frac{\tau}{12} \iota(X) \wedge \iota(Y),
$$

where $\iota$ is the duality between the tangent bundle and the cotangent bundle of $M$ by means of the metric $g$. Let $\left\{e_{1}, e_{2}=J e_{1}, e_{3}, e_{4}=J e_{3}\right\}$ be a (local) unitary frame field and put $e^{i}=\iota\left(e_{i}\right)$. Then, the Kähler form is represented by $\Omega=-e^{1} \wedge e^{2}-e^{3} \wedge e^{4}$. Further, 
we see that

$$
\begin{gathered}
\{\Phi, J \Phi\}=\left\{\frac{1}{\sqrt{2}}\left(e^{1} \wedge e^{3}-e^{2} \wedge e^{4}\right), \frac{1}{\sqrt{2}}\left(e^{1} \wedge e^{4}+e^{2} \wedge e^{3}\right)\right\} \\
\left\{\Psi_{1}, \Psi_{2}, \Psi_{3}\right\}=\left\{\frac{1}{\sqrt{2}}\left(e^{1} \wedge e^{2}-e^{3} \wedge e^{4}\right), \frac{1}{\sqrt{2}}\left(e^{1} \wedge e^{3}+e^{2} \wedge e^{4}\right), \frac{1}{\sqrt{2}}\left(e^{1} \wedge e^{4}-e^{2} \wedge e^{3}\right)\right\}
\end{gathered}
$$

are (local) orthonormal bases of $L M$ and $\wedge_{0}^{1,1} M=\wedge_{-}^{2} M$, respectively.

In this paper, for any orthonormal basis (resp., any local orthonormal frame field) $\left\{e_{1}, e_{2}, e_{3}, e_{4}\right\}$ of a point $p \in M$ (resp., on a neighborhood of $p$ ), we will adopt the following notational convention:

$$
\begin{gathered}
J_{i j}=g\left(J e_{i}, e_{j}\right), \quad \Gamma_{i j k}=g\left(\nabla_{e_{i}} e_{j}, e_{k}\right), \\
R_{i j k l}=g\left(R\left(e_{i}, e_{j}\right) e_{k}, e_{l}\right), \ldots, R_{\bar{i} \bar{j} \bar{k} \bar{l}}=g\left(R\left(J e_{i}, J e_{j}\right) J e_{k}, J e_{l}\right), \\
\rho_{i j}=\rho\left(e_{i}, e_{j}\right), \ldots, \rho_{\bar{i} \bar{j}}=\rho\left(e_{\bar{i}}, e_{\bar{j}}\right), \\
\rho_{i j}^{*}=\rho^{*}\left(e_{i}, e_{j}\right), \ldots, \rho_{\bar{i} \bar{j}}^{*}=\rho^{*}\left(e_{\bar{i}}, e_{\bar{j}}\right), \\
\nabla_{i} J_{j k}=g\left(\left(\nabla_{e_{i}} J\right) e_{j}, e_{k}\right), \ldots, \nabla_{\bar{i}} J_{\bar{j} \bar{k}}=g\left(\left(\nabla_{e_{\bar{i}}} J\right) e_{\bar{j}}, e_{\bar{k}}\right),
\end{gathered}
$$

and so on, where the Latin indices run over the range $1,2,3,4$. We define functions $A$, $B, C, D, G$, and $K$ on $M$ by

$$
\begin{aligned}
& A=\sum_{i, j, k, l, a=1}^{4}\left(\nabla_{a} J_{i j}\right) R_{i j k l}\left(\nabla_{a} J_{k l}\right), \\
& B=\sum_{i, j, k, l, a=1}^{4}\left(\nabla_{a} J_{i j}\right)\left(\nabla_{a} J_{k l}\right)\left(\nabla_{b} J_{i j}\right)\left(\nabla_{b} J_{k l}\right), \\
& C=\sum_{i, j, k, l=1}^{4} R_{i j k l} R_{i j k \bar{l}}, \quad D=\sum_{i, j, k, l=1}^{4}\left(R_{i j k l}-R_{i j \bar{k} \bar{l}}\right)^{2}, \\
& G=\sum_{i, j=1}^{4}\left(\rho_{i j}^{*}-\rho_{j i}^{*}\right)^{2}, \quad K=(u-v)^{2}+4 w^{2},
\end{aligned}
$$

where $u=g(\Re(\Phi), \Phi), v=g(\mathscr{R}(J \Phi), J \Phi)$, and $w=g(\mathscr{R}(\Phi), J \Phi)$. First, we will prove the following.

LEMMA 2.1. The norm of the self-dual Weyl operator ${ }^{W^{+}}{ }^{+}$is given by

$$
\left\|W^{+}\right\|^{2}=\frac{1}{16}\left(G+D+\left(\tau^{*}\right)^{2}-\frac{\tau^{2}}{3}\right)
$$

Proof. Let $\left\{e_{1}, e_{2}=J e_{1}, e_{3}, e_{4}=J e_{3}\right\}$ be any (local) unitary frame field on $M$ and we put $\Omega_{0}=-\Omega / \sqrt{2}=\left(e^{1} \wedge e^{2}+e^{3} \wedge e^{4}\right) / \sqrt{2}, \Phi=\left(e^{1} \wedge e^{3}-e^{2} \wedge e^{4}\right) / \sqrt{2}$, and $J \Phi=$ $\left(e^{1} \wedge e^{4}+e^{2} \wedge e^{3}\right) / \sqrt{2}$. Then, $\left\{\Omega_{0}, \Phi, J \Phi\right\}$ is an orthonormal basis of $\wedge_{+}^{2} M$. Thus, we have

$$
\begin{aligned}
\left\|W^{+}\right\|^{2}= & g\left(\mathcal{W}^{+}\left(\Omega_{0}\right), \Omega_{0}\right)^{2}+g\left(\mathcal{W}^{+}\left(\Omega_{0}\right), \Phi\right)^{2}+g\left(W^{+}\left(\Omega_{0}\right), J \Phi\right)^{2} \\
& +g\left(W^{+}(\Phi), \Omega_{0}\right)^{2}+g\left(\mathcal{W}^{+}(\Phi), \Phi\right)^{2}+g\left(W^{+}(\Phi), J \Phi\right)^{2} \\
& +g\left(W^{+}(J \Phi), \Omega_{0}\right)^{2}+g\left(W^{+}(J \Phi), \Phi\right)^{2}+g\left(\mathcal{W}^{+}(J \Phi), J \Phi\right)^{2} .
\end{aligned}
$$


Taking account of (2.4), we have

$$
\begin{aligned}
g\left(W^{+}\left(\Omega_{0}\right), \Omega_{0}\right) & =\frac{1}{2}\left(-R_{1212}-2 R_{1234}-R_{3434}-\frac{\tau}{6}\right)=\frac{1}{12}\left(3 \tau^{*}-\tau\right), \\
g\left(W^{+}\left(\Omega_{0}\right), \Phi\right) & =\frac{1}{2}\left(-R_{1213}-R_{1224}-R_{3413}-R_{3424}\right)=-\frac{1}{2}\left(\rho_{14}^{*}-\rho_{41}^{*}\right), \\
g\left(W^{+}\left(\Omega_{0}\right), J \Phi\right) & =\frac{1}{2}\left(-R_{1214}-R_{1223}-R_{3414}-R_{3423}\right)=\frac{1}{2}\left(\rho_{13}^{*}-\rho_{31}^{*}\right), \\
g\left(W^{+}(\Phi), \Phi\right) & =\frac{1}{2}\left(-R_{1313}+2 R_{1324}-R_{2424}-\frac{\tau}{6}\right)=-\left(R_{1313}-R_{1324}\right)-\frac{\tau}{12}, \\
g\left(W^{+}(\Phi), J \Phi\right) & =\frac{1}{2}\left(-R_{1314}-R_{1323}+R_{2414}+R_{2423}\right)=-\left(R_{1314}+R_{1323}\right), \\
g\left(W^{+}(J \Phi), J \Phi\right) & =\frac{1}{2}\left(-R_{1414}-2 R_{1423}-R_{2323}-\frac{\tau}{6}\right)=-\left(R_{1414}+R_{1423}\right)-\frac{\tau}{12}
\end{aligned}
$$

Thus, we have

$$
\begin{aligned}
\left\|W^{+}\right\|^{2}= & \frac{1}{12^{2}}\left(3 \tau^{*}-\tau\right)^{2}+\frac{2 \tau^{2}}{12^{2}}+\frac{1}{2}\left(\rho_{13}^{*}-\rho_{31}^{*}\right)^{2}+\frac{1}{2}\left(\rho_{14}^{*}-\rho_{41}^{*}\right)^{2} \\
& +\left(R_{1313}-R_{1324}\right)^{2}+\left(R_{1314}+R_{1323}\right)^{2}+\left(R_{1314}+R_{1323}\right)^{2} \\
& +\left(R_{1414}+R_{1423}\right)^{2}+\frac{\tau}{6}\left(R_{1313}-R_{1324}+R_{1414}+R_{1423}\right) \\
= & \frac{1}{12^{2}}\left(3 \tau^{*}-\tau\right)^{2}+\frac{2 \tau^{2}}{12^{2}}+\frac{G}{8} \\
& +\frac{1}{4} \sum_{i<j, k<l}\left(R_{i j k l}-R_{i j \bar{k} \bar{l}}\right)^{2}-\frac{1}{4} \sum_{k<l}\left(R_{12 k l}-R_{12 \bar{k} \bar{l}}\right)^{2} \\
& -\frac{1}{4} \sum_{k<l}\left(R_{34 k l}-R_{34 \bar{k} \bar{l}}\right)^{2}+\frac{\tau}{6}\left(-\frac{\tau}{4}-R_{1212}-R_{1234}\right) \\
= & \frac{1}{12^{2}}\left(3 \tau^{*}-\tau\right)^{2}+\frac{2 \tau^{2}}{12^{2}}+\frac{G}{8}+\frac{D}{16}-\frac{G}{32}-\frac{G}{32}+\frac{\tau}{6}\left(-\frac{\tau}{4}+\frac{\tau^{*}}{4}\right) \\
= & \frac{D}{16}+\frac{G}{16}+\frac{\left(\tau^{*}\right)^{2}}{16}-\frac{\tau^{2}}{48} .
\end{aligned}
$$

The lemma follows.

Next, we recall the following equalities established in [6]:

$$
\begin{gathered}
A=\frac{1}{4} B=\frac{\left(\tau^{*}-\tau\right)^{2}}{2}, \\
C=-2 K+\frac{\left(\tau^{*}-\tau\right)^{2}}{8}, \\
G=4\left\|\rho^{*}\right\|^{2}-\left(\tau^{*}\right)^{2}=16\left\{\left(\rho_{13}^{*}\right)^{2}+\left(\rho_{14}^{*}\right)^{2}\right\}, \\
K=(u+v)^{2}+4\left(w^{2}-u v\right)=\frac{\left(\tau^{*}-\tau\right)^{2}}{16}-4 \operatorname{det} \mathscr{R}_{L M}^{\prime}, \\
\left\|\mathscr{R}_{L M}\right\|^{2}=\frac{1}{16} D, \quad\left\|\mathscr{R}_{L M}^{\prime}\right\|^{2}=\frac{1}{16}(D-G),
\end{gathered}
$$


where $\mathscr{R}_{L M}$ is the restriction of $\mathscr{R}$ to $L M$ and $\mathscr{R}_{L M}^{\prime}=P_{L M} \circ \mathscr{R}_{L M}$, the composition of $\mathscr{R}_{L M}$ and the natural projection $P_{L M}: \wedge^{2} M \rightarrow L M$. We define a vector field $\eta=\left(\eta_{a}\right)$ on $M$ by $\eta_{a}=\sum_{i, j=1}^{4}\left(\nabla_{a} J_{i j}\right) \rho_{i \bar{j}}^{*}$, then we obtain the following (see [6, (2.23)]):

$$
\Delta \tau^{*}=\frac{G}{2}+4 K+\frac{\left(3 \tau^{*}-\tau\right)\left(\tau^{*}-\tau\right)}{4}-4 \operatorname{div} \eta
$$

Further, from (2.12) and the curvature identity

$$
R_{i j k l}-R_{i j \bar{k} \bar{l}}-R_{\bar{i} \bar{j} k l}+R_{\bar{i} \bar{j} \bar{k} \bar{l}}+R_{\bar{i} j \bar{k} l}+R_{\bar{i} j k \bar{l}}+R_{i \bar{j} \bar{k} l}+R_{i \bar{j} k \bar{l}}=2 \sum_{a=1}^{4}\left(\nabla_{a} J_{i j}\right) \nabla_{a} J_{k l}
$$

by Gray [3] for almost Kähler manifold, we have

$$
\begin{aligned}
A & =\frac{1}{2} \sum R_{i j k l}\left(R_{i j k l}-R_{i j \bar{k} \bar{l}}-R_{\bar{i} \bar{j} k l}+R_{\bar{i} \bar{j} \bar{k} \bar{l}}+R_{\bar{i} \bar{j} \bar{k} l}+R_{\bar{i} j k \bar{l}}+R_{i \bar{j} \bar{k} l}+R_{i \bar{j} k \bar{l}}\right) \\
& =\frac{1}{4} \sum\left(R_{i j k l}-R_{i j \bar{k} \bar{l}}\right)^{2}-\frac{1}{4} \sum\left(R_{i j k l}-R_{i j \bar{k} \bar{l}}\right)\left(R_{\bar{i} \bar{j} k l}-R_{\bar{i} \bar{j} \bar{k} \bar{l}}\right)+2 \sum R_{i j k l} R_{i \bar{j} k \bar{l}} \\
& =\frac{D}{4}-\frac{1}{4}\left\{-16\left\|\Re_{L M}^{\prime}\right\|^{2}+\sum\left(R_{i j 12}+R_{i j 34}-R_{\bar{i} \bar{j} 12}-R_{\bar{i} \bar{j} 34}\right)^{2}\right\}+2 C \\
& =\frac{D}{4}+4\left\|\Re_{L M}^{\prime}\right\|^{2}-\frac{G}{4}+2 C \\
& =\frac{D}{2}-\frac{G}{2}-4 K+\frac{\left(\tau^{*}-\tau\right)^{2}}{4} .
\end{aligned}
$$

Thus, from (2.12) and this equality, we obtain

$$
\frac{D}{2}-\frac{G}{2}-4 K-\frac{\left(\tau^{*}-\tau\right)^{2}}{4}=0
$$

Now, we are ready to prove the following.

THEOREM 2.2. Let $M=(M, J, g)$ be a four-dimensional compact almost Kähler-Einstein manifold with negative scalar curvature. If $M$ satisfies

$$
\int_{M}\left\{G+\tau\left(\tau^{*}-\tau\right)\right\} d V \geq 0
$$

or, more strictly, if $\tau^{*}-\tau \leq-G / \tau$ at each point of $M$, then $M$ is Kähler-Einstein.

Proof. From (2.8), we have

$$
24 \int_{M}\left\|W^{+}\right\|^{2} d V-\int_{M} \tau^{2} d V=\frac{3}{2} \int_{M}\left\{G+D+\left(\tau^{*}-\tau\right)\left(\tau^{*}+\tau\right)\right\} d V
$$

On one hand, from (2.13) and (2.16), we have

$$
0=\int_{M}\left\{\frac{G}{2}+4 K+\frac{\left(3 \tau^{*}-\tau\right)\left(\tau^{*}-\tau\right)}{4}\right\} d V=\int_{M}\left\{\frac{D}{2}+\frac{\tau^{*}\left(\tau^{*}-\tau\right)}{2}\right\} d V
$$


Thus, from (2.18) and (2.19), we obtain

$$
24 \int_{M}\left\|W^{+}\right\|^{2} d V-\int_{M} \tau^{2} d V=\frac{2}{3} \int_{M}\left\{G+\tau\left(\tau^{*}-\tau\right)\right\} d V .
$$

Therefore, from Corollary 1.3, the assertion of the theorem immediately follows.

REMARK 2.3. The above theorem is concerned with the following facts.

(1) For a compact four-dimensional almost Kähler-Einstein manifold, the function $\tau^{*}-\tau$ vanishes at some point of $M$ (see $\left.[1,5]\right)$.

(2) A four-dimensional compact almost Kähler-Einstein and weakly $*$-Einstein manifold $(G \equiv 0)$ is a Kähler manifold (see [7]).

(3) Let $M$ be a four-dimensional compact strictly almost Kähler-Einstein, but not weakly $*$-Einstein manifold. Then, we see that $G>0$ on $M_{0}=\left\{p \in M \mid \tau^{*}-\tau>\right.$ 0 , and hence $\tau^{*}-\tau=0$ at which $G=0$ (see [5]).

\section{REFERENCES}

[1] J. Armstrong, On four-dimensional almost Kähler manifolds, Quart. J. Math. Oxford Ser. (2) 48 (1997), no. 192, 405-415.

[2] S. I. Goldberg, Integrability of almost Kaehler manifolds, Proc. Amer. Math. Soc. 21 (1969), 96-100.

[3] A. Gray, Curvature identities for Hermitian and almost Hermitian manifolds, Tôhoku Math. J. (2) 28 (1976), no. 4, 601-612.

[4] M. Itoh, Almost Kähler 4-manifolds, $L^{2}$-scalar curvature functional and Seiberg-Witten equation, to appear in Internat. J. Math.

[5] T. Oguro and K. Sekigawa, Notes on strictly almost Kähler manifolds of dimension four, to appear in Yokohama Math. J.

[6] __ On some four-dimensional almost Kähler Einstein manifolds, Kodai Math. J. 24 (2001), no. 2, 226-258.

[7] T. Oguro, K. Sekigawa, and A. Yamada, Four-dimensional almost Kähler Einstein and weakly *-Einstein manifolds, Yokohama Math. J. 47 (1999), no. 1, 75-92.

[8] K. Sekigawa, On some compact Einstein almost Kähler manifolds, J. Math. Soc. Japan 39 (1987), no. 4, 677-684.

R. S. Lemence: Department of Mathematical Science, Graduate School of Science and Technology, Niigata University, Niigata 950-2181, Japan

E-mail address: f02n406n@mai1.cc.ni igata-u.ac.jp

T. Oguro: Department of Mathematical Sciences, School of Science and Engineering, Tokyo Denki University, Saitama 350-0394, Japan

E-mail address: oguro@r.dendai .ac.jp

K. Sekigawa: Department of Mathematics, Faculty of Science, Niigata University, Niigata 9502181, Japan

E-mail address: sekigawa@sc.ni igata-u.ac.jp 


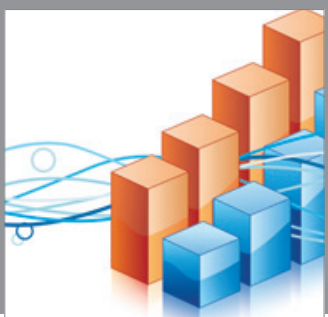

Advances in

Operations Research

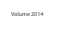

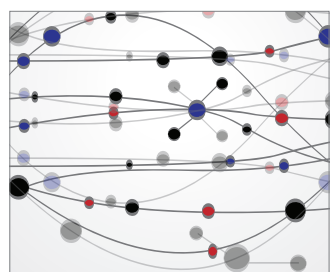

\section{The Scientific} World Journal
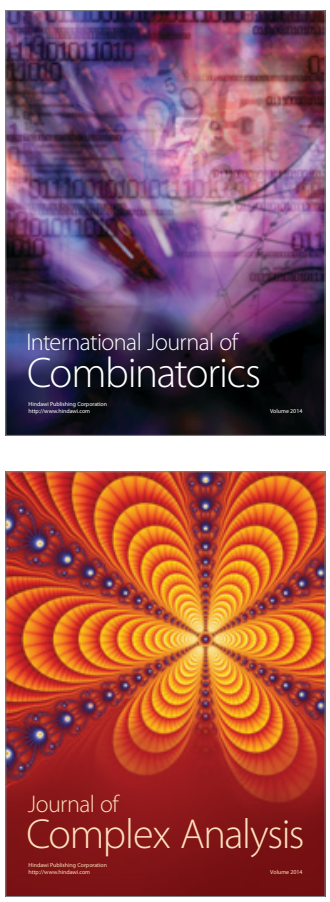

International Journal of

Mathematics and

Mathematical

Sciences
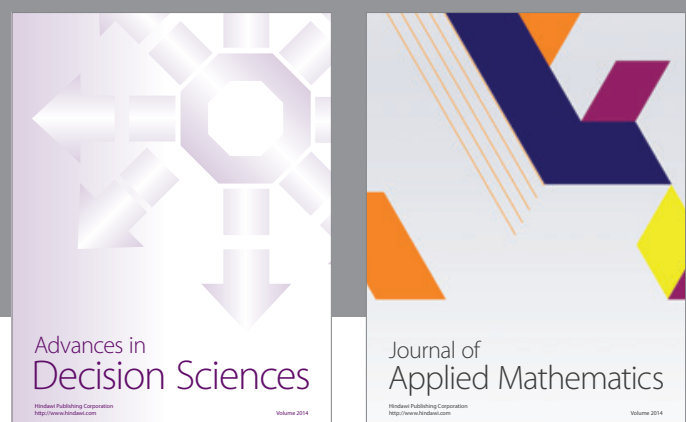

Journal of

Applied Mathematics
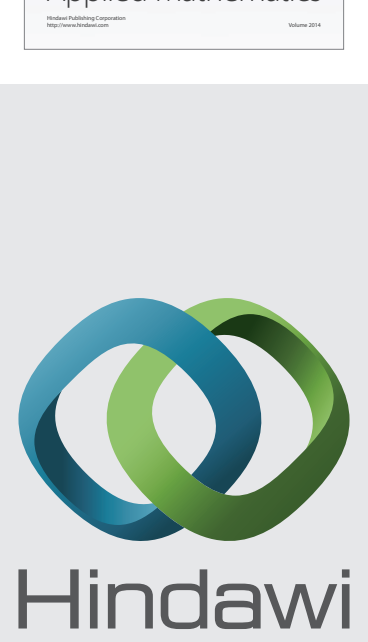

Submit your manuscripts at http://www.hindawi.com
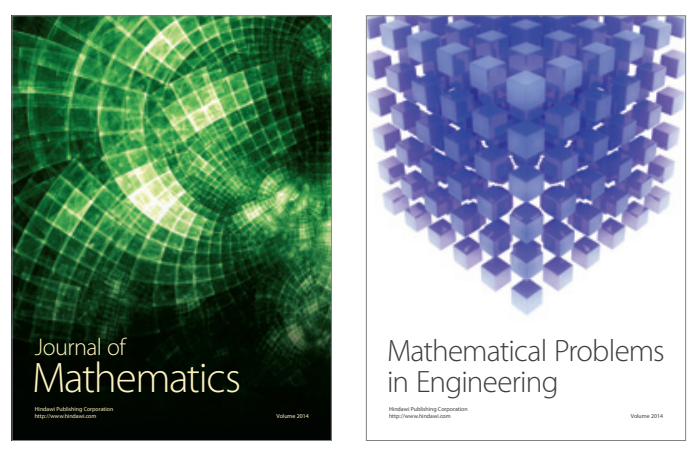

Mathematical Problems in Engineering
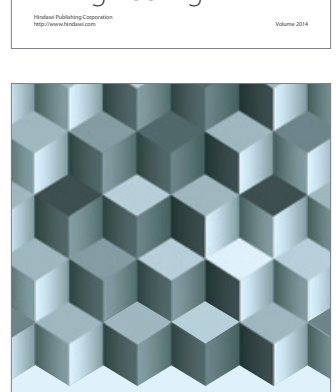

Journal of

Function Spaces
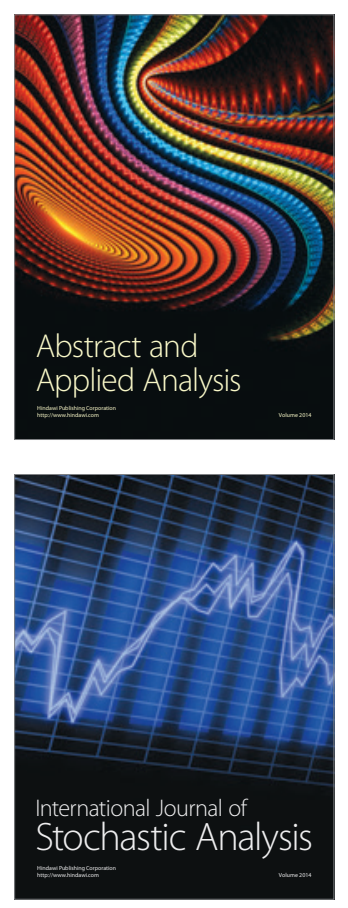

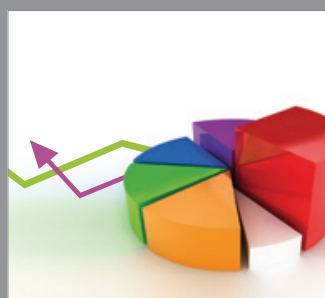

ournal of

Probability and Statistics

Promensencen
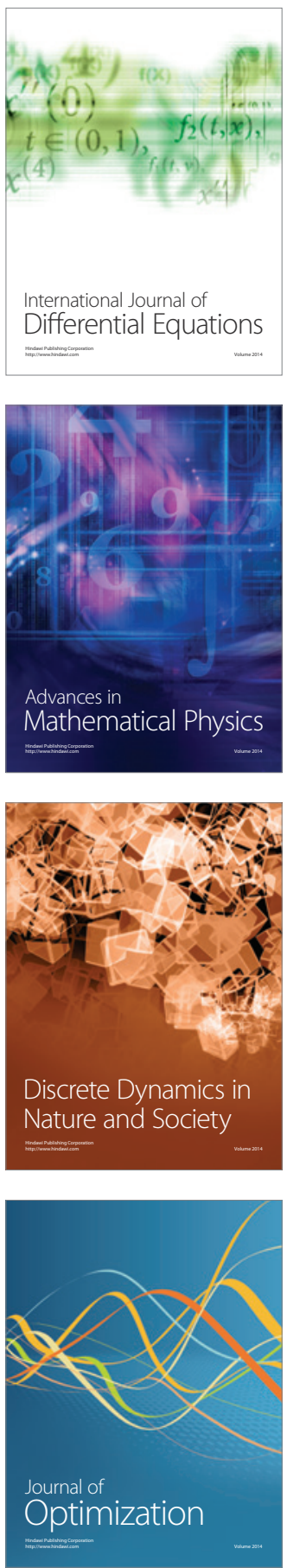